\title{
Is Low-Grade Intraventricular Hemorrhage in Very Preterm Infants an Innocent Condition? Structural and Functional Evaluation of the Brain Reveals Regional Neurodevelopmental Abnormalities
}

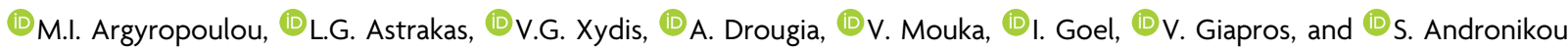

\begin{abstract}
BACKGROUND AND PURPOSE: There is increasing evidence of abnormal neurodevelopmental outcomes in very preterm infants with low-grade intraventricular hemorrhage grades I and II. Our purpose was to evaluate the effects of low-grade intraventricular hemorrhage on gray and white matter integrity.
\end{abstract}

\begin{abstract}
MATERIALS AND METHODS: MR imaging at around term-equivalent age was performed in 16 very preterm infants (mean gestational age, $28.8 \pm 5.3$ weeks) with mild intraventricular hemorrhage on brain sonography and 13 control subjects (mean gestational age, $29.6 \pm 4.1$ weeks) without intraventricular hemorrhage. Structural and functional evaluation of the cortex was performed using regional measurements of surface area, thickness and volume, and resting-state fMRI, respectively, and of WM microstructural integrity, applying Tract-Based Spatial Statistics to diffusion tensor imaging data.
\end{abstract}

RESULTS: Compared with the control infants, the infants with low-grade intraventricular hemorrhage had decreases in the following: 1) GM surface area in Brodmann areas 19 left and 9 and 45 right, and GM volume in Brodmann areas 9 and 10 right; 2) fractional anisotropy bilaterally in major WM tracts; and 3) brain activity in the left lower lateral and in the right higher medial somatosensory cortex.

CONCLUSIONS: Very premature infants with low-grade intraventricular hemorrhage at around term-equivalent age may present with regional abnormalities, appearing on imaging studies as cortical underdevelopment, functional impairment, and microstructural immaturity of major WM tracts.

ABBREVIATIONS: BA = Brodmann area; BOLD = blood oxygen level-dependent; FA = fractional anisotropy; fALFF = fractional amplitude of low-frequency fluctuations; $\mathrm{GA}=$ gestational age; GABA = gamma-aminobutyric acid; GMH = germinal matrix hemorrhage; IVH = intraventricular hemorrhage; PALSBI2 = Population-Average, Landmark- and Surface-Based atlas; SSC = somatosensory cortex

G erminal matrix hemorrhage (GMH) is hemorrhage in the ganglionic eminence, which is the longest lasting germinal ventricular/subventricular zone, producing precursors of neurons, of oligodendrocytes, and of astrocytes. ${ }^{1}$ GMH may rupture through the ventricular ependyma and evolve into intraventricular hemorrhage (IVH). The severity of GMH-IVH has been graded according to the Papile classification system, in which grade I is GMH alone, grade II is IVH occupying $<50 \%$ of the

Received October 20, 2019; accepted after revision January 4, 2020.

From the Departments of Radiology (M.I.A., V.G.X., V.M., I.G.), and Medical Physics (L.G.A.), and Neonatology Unit (A.D., V.G., S.A.), Faculty of Medicine, University of loannina, loannina, Greece.

Please address correspondence to Maria I. Argyropoulou, MD, Department of Radiology, Faculty of Medicine, School of Health Sciences, University of Ioannina, PO Box 1186, 45110 loannina, Greece; e-mail: margyrop@uoi.gr

Indicates article with supplemental on-line tables.

http://dx.doi.org/10.3174/ajnr.A6438 lateral ventricle, grade III is IVH occupying $>50 \%$ of the lateral ventricle, and grade IV is grade III associated with periventricular venous infarction. ${ }^{2}$ GMH-IVH is one of the most common neuropathologic conditions of prematurity, presenting increasing incidence with decreasing gestational age (GA) and birth weight. ${ }^{3-5}$ In very preterm infants (GA, $<32$ gestational weeks; birth weight, $<1500 \mathrm{~g}$ ), the incidence of GMH-IVH is $25 \%$ $30 \%$, and this number remains stable despite advances in neonatal intensive care. ${ }^{3,5}$ Severe IVH (grades III and IV) is associated with adverse neurodevelopmental outcomes, including motor dysfunction and cerebral palsy, impairment of high-level cognitive functions, and visual and hearing disturbances. ${ }^{3,6}$ Destruction of the periventricular WM due to hydrocephalus and periventricular venous infarction has been suggested as the main cause of these morbidities. ${ }^{3,6}$

Low-grade IVH (grades I and II) had long been considered free of risk of adverse neurodevelopmental outcomes, but new 
evidence is challenging this belief. ${ }^{3,6}$ Abnormal neurodevelopmental outcomes have also been recently reported in infants with low-grade IVH, though to a lesser extent and severity than in those with severe IVH. ${ }^{3}$ During the peak period of vulnerability for GMH-IVH (gestational weeks 25-32), major developmental events are taking place, including generation of late-migrating gamma-aminobutyric acid (GABA)ergic interneurons, critical for the development of the cortex and thalamus, and of oligodendroglial precursor cells, which give rise to premyelinating oligodendrocytes, along with rapid axonal growth and elongation. ${ }^{1,5,7}$ An increasing body of evidence shows that GMH-IVH may intervene in these events, arresting germ cell proliferation and impairing cell differentiation and migration and axonal growth. ${ }^{5,6,8-10}$

The period between 28 and 40 gestational weeks is crucial for structural and functional development of the brain, and MR imaging evaluation at around term-equivalent age could be a useful means of assessing the effects of GMH-IVH. MR imaging offers powerful tools for evaluating maturational structural and functional integrity of the brain. Gyration has been associated with structural connectivity and can be evaluated at the regional level by voxel wise measurements of the cortical surface area, thickness, and volume. ${ }^{11}$ Functional connectivity of the brain can be assessed by resting-state fMRI, which detects low-frequency $(0.01-0.10 \mathrm{~Hz})$ fluctuations of the blood oxygen leveldependent (BOLD) signal in specific anatomic areas. Anatomic areas presenting with temporal correlations in these fluctuations are considered functionally connected and form a network. ${ }^{12}$ WM integrity can be evaluated with DTI, and one of the most frequently used methods for voxel wise assessment is Tract-Based Spatial Statistics (TBSS; http://fsl.fmrib.ox.ac.uk/ fsl/fslwiki/TBSS). ${ }^{12}$ The few MR imaging studies on the effect of low-grade IVH on brain structure have reported decreased total GM volume and microstructural abnormalities of major WM tracts. ${ }^{13,14}$ To our knowledge, no study evaluating the effect of GMH-IVH on functional connectivity of the brain has been published.

Because structural and functional maturation of the brain are interrelated, we considered it useful to evaluate both in a series of very premature infants with low-grade IVH, in comparison with premature control subjects without IVH. We hypothesized that low-grade IVH may intervene in the development of GM and WM, affecting the microstructure and function of the brain. We assessed changes in structural and functional maturation of the cortex using voxel wise regional measurements of surface area, thickness, and volume and resting-state fMRI, respectively, and WM microstructural integrity by applying TBSS to DTI data.

\section{MATERIALS AND METHODS Study Population}

Very preterm neonates, born between January 2014 and May 2017 and admitted to the neonatal intensive care unit of the University Hospital of Ioannina, were evaluated with MR imaging at around term-equivalent age. After we applied the exclusion criteria (congenital malformations; syndromes; congenital TORCH [toxoplasmosis, other agents, ie, syphilis, varicella-zoster, parvovirus B19, rubella, cytomegalovirus, and herpes simplex infections) infection; punctuate WM hyperintensities; periventricular leuko- malacia; high-grade IVH; infarction; stroke; and inappropriate quality of the MR imaging examination), the study population consisted of 16 premature infants with low-grade IVH on brain ultrasound and MR imaging and 13 control subjects with normal brain ultrasound and MR imaging findings. All infants included in the final study population had been discharged at 36 weeks' postmenstrual age or with 1800-g body weight and were free of problems. The study was performed with the approval of the institutional review board, and the parents of all the infants eligible to participate in the study provided their informed written consent for inclusion of their infants in the study.

\section{MR Imaging Protocol}

MR imaging was performed using an Intera 1.5T MR imaging scanner (Philips Healthcare, Best, the Netherlands). All examinations were performed with the infant under sedation with oral chloral hydrate $(20-30 \mathrm{mg} / \mathrm{Kg})$. The imaging protocol comprised anatomic MR imaging, resting-state fMRI, and DTI. Anatomic MR imaging was performed with a T1-weighted high-resolution 3D spoiled gradient-echo sequence (TR/TE/flip angle, $25 \mathrm{~ms} /$ $4.6 \mathrm{~ms} / 6^{\circ}$; voxel size, $\left.0.86 \times 0.86 \times 1 \mathrm{~mm}^{3}\right)$ and T2-weighted sequences (TR/TE, 3500/90 ms; section thickness, $2.5 \mathrm{~mm}$; section gap, $0 \mathrm{~mm}$ ). A single-shot echo-planar sequence was used in all resting-state $\mathrm{fMRI}$ imaging scans, with the following parameters: TR/TE/flip angle, $2000 \mathrm{~ms} / 40 \mathrm{~ms} / 90^{\circ}$; number of signal averages, 1; dynamic scans, 200 real +2 dummy scans; voxel size, $3.4 \times$ $3.4 \times 4 \mathrm{~mm}^{3}$. DTI was performed with a single-shot spin-echo echo-planar sequence with the following parameters: TR/TE, 9807/131 ms; voxel size, $1.8 \times 1.8 \times 3 \mathrm{~mm}^{3}$; maximum b-value, $700 \mathrm{~s} / \mathrm{mm}^{2} ; 16$ noncolinear diffusion directions; number of signal intensity averages, 2 .

\section{Image Analyses}

Brain Surface Analysis. Cortical surface analysis of the T1weighted images was performed using a FreeSurfer (http:// surfer.nmr.mgh.harvard.edu) image-processing pipeline developed especially for infant clinical MR images. ${ }^{15}$ The pipeline consists of 3 steps: skull-stripping, brain segmentation, and surface extraction. Surface analysis was provided for each subject and for each hemisphere map of cortical thickness and cortical area. Group comparison of each map was performed at the ROI level after parcellating each brain using the Brodmann Population-Average, Landmark- and Surface-Based (PALSB12) atlas. ${ }^{16}$ Area, thickness, volume, and curvature in the Brodmann areas (BAs) of the surface maps were compared between neonates with and without IVH using the Mann-Whitney $U$ test combined with a Bonferonni correction for multiple comparison differences (SPSS, Version 21; IBM, Armonk, New York).

White Matter Microstructural Analysis. Images were processed using the FMRIB Software Library (FSL; http://www.fmrib.ox.ac. uk/fsl). Using the FMRIB Diffusion Toolbox (http://fsl.fmrib.ox. ac.uk/fsl/fslwiki/FDT) for each subject, we performed the following steps: 1) All images were corrected for distortion and subject motion effects; 2) the skull was extracted using the Brain Extraction Tool (http://fsl.fmrib.ox.ac.uk/fsl/fslwiki/BET); 3) a diffusion tensor model was fitted to the data and produced maps 


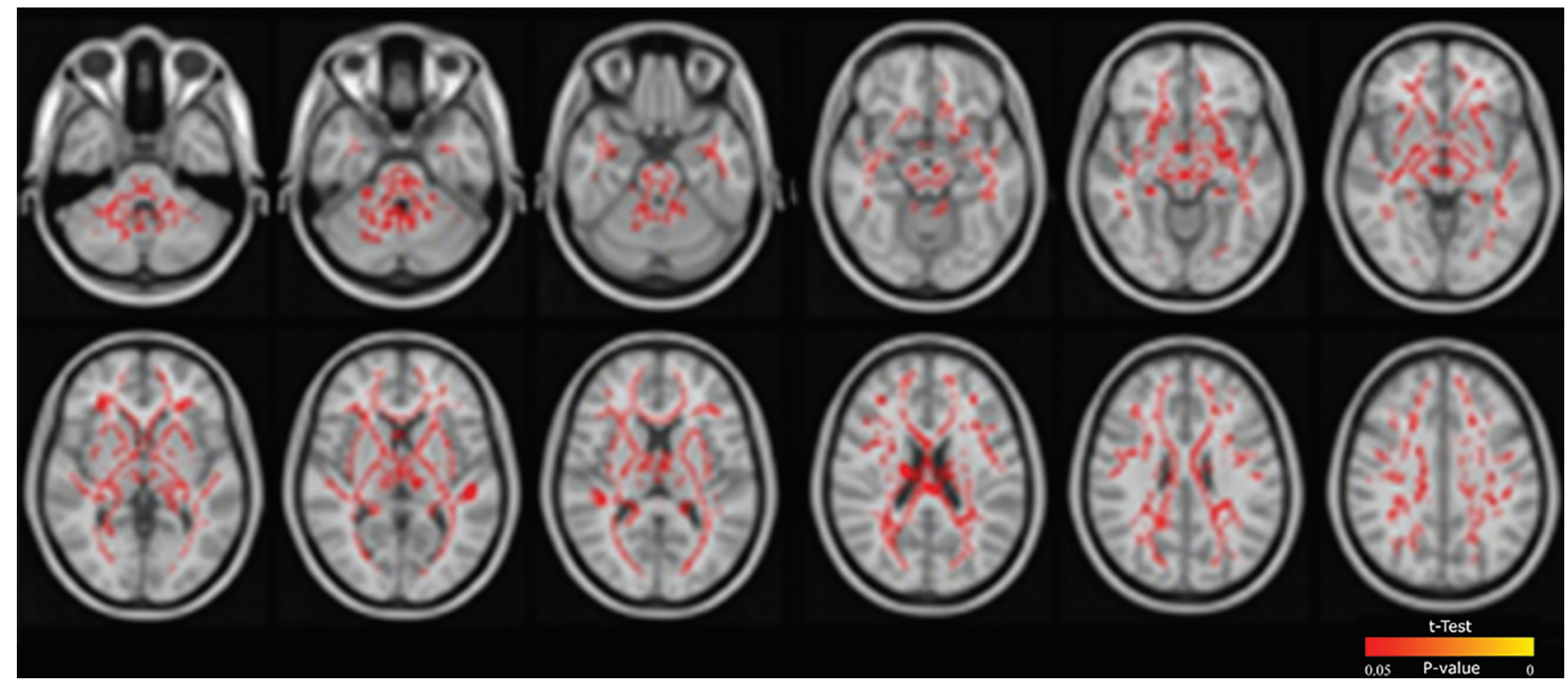

FIG 1. Low-grade IVH in very premature infants: white matter areas (in red) with reduced FA overlaid on a template T1-weighted image.

of fractional anisotropy (FA), measuring the degree of directionality of WM tracts, mean diffusivity, radial diffusivity measuring diffusion perpendicular to the WM tracts, and axial diffusivity measuring diffusion parallel to the WM tracts; 4) FA images were aligned to a target in a common space using an optimized TBSS protocol for neonates; ${ }^{17}$ 5) a mean FA map was created and thinned $(\mathrm{FA}>0.15)$ to create a mean FA skeleton; and 6) aligned FA, axial diffusivity, and radial diffusivity data were projected onto the mean FA skeleton. Differences in FA, mean diffusivity, radial diffusivity, and axial diffusivity between neonates with and without IVH were assessed using voxel wise statistics, based on a permutation-based inference with 5000 permutations. The results were corrected for multiple comparisons by controlling the family-wise error rate following threshold-free cluster enhancement, with a threshold of $P<.05$. The labeling of the significant clusters was based on the Johns Hopkins University white matter tractography atlas.

fMRI Analysis. Functional connectivity was assessed using the CONN toolbox (https://web.conn-toolbox.org/). Preprocessing steps were applied to correct for head movement and differences in section timing. Functional images were registered to high-resolution anatomic images and normalized and smoothed with a Gaussian kernel of $6-\mathrm{mm}$ full width at half maximum. The Template-O-Matic toolbox (https://irc.cchmc.org/software/tom. php) was used for the normalization step. Physiologic and movement components in the BOLD signal were removed using the Component Based Noise Correction Method (CompCor; https:// www.ncbi.nlm.nih.gov/pmc/articles/PMC2214855).

Slow fluctuations in activity are a fundamental feature of the resting brain and can be used to determine correlated activity between brain regions and to define resting-state networks. The relative magnitude of these fluctuations can differ between brain regions and between subjects and thus may act as a marker of individual differences or dysfunction. The fractional amplitude of low-frequency fluctuations (fALFF) is a measure that quantifies these low-frequency oscillations. The fALFF was calculated at each individual voxel as the relative amplitude (root mean square ratio) of the BOLD signal fluctuations at the frequency band of $0.008-0.09 \mathrm{~Hz}$, compared with the entire frequency band. ${ }^{18}$ Group analysis of fALFF maps was performed using a $t$ test at the voxel level. The $t$ maps were thresholded using a combination of height threshold ( $P=.001$ uncorrected) and cluster extent threshold ( $P=.05$, family-wise error corrected).

\section{RESULTS}

None of the studied infants presented with necrotic enterocolitis or retinopathy of prematurity. There was no significant difference between infants with low-grade IVH and the control subjects in GA, birth weight, postmenstrual age at MR imaging, body weight at MR imaging, antenatal steroid administration, duration of mechanical ventilation, infection, patent ductus arteriosus, or chronic lung disease (On-Table 1).

\section{Brain Surface Analysis}

ROI surface analysis, based on the FreeSurfer PALSB12 atlas, showed that low-grade IVH affects BAs 19, 9, 45, and 10 (On-line Tables 2-9). Specifically, it reduced the volume of BAs 9 and 10 in the right hemisphere (On-line Table 9), the area of BAs 9 and 45 in the right hemisphere (On-line Table 3), and the area of Brodmann area 19 in the left hemisphere (On-line Table 2).

\section{White Matter Microstructural Analysis}

TBSS analysis revealed that low-grade IVH produces an extensive decrease in FA in multiple WM tracts, including the anterior thalamic radiation, corticospinal tract, cingulum, forceps minor and major, inferior fronto-occipital fasciculus, inferior and superior longitudinal fasciculus, and uncinate fasciculus (On-line Table 10 and Fig 1). The other diffusion metrics remained unaffected.

\section{fMRI Analysis}

fALFF analysis showed decreased brain activity in the resting state of the infants with low-grade IVH in the left lower lateral 


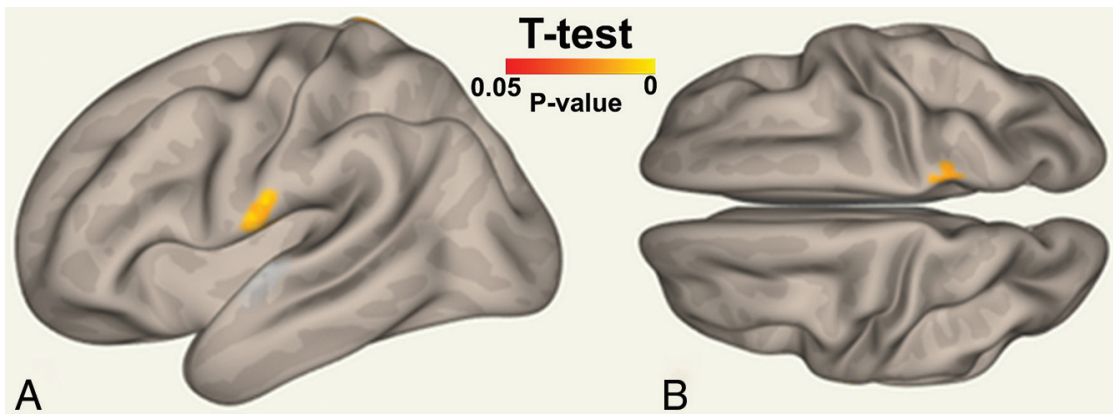

FIG 2. Brain area with decreased fALFF (yellow) in the left lower lateral (A) and right higher medial (B) somatosensory cortex, overlaid on a Montreal Neurological Institute template, of very premature infants with low-grade IVH.

and the right higher medial somatosensory cortex (Fig 2 and Online Table 11).

\section{DISCUSSION}

The primary goal of this study was to evaluate, in very preterm infants, the effects of low-grade IVH on brain structure and function. Analysis of DTI data for WM microstructural changes was performed with TBSS, which offers advanced registration capabilities and robust nonparametric assessment of local differences between groups. ${ }^{19}$ The cortical structure was studied with FreeSurfer, a state-of-the-art software capable of quantifying thickness, area, and curvature, not available with standard volumetric analysis. fMRI analysis was performed with fALFF, which is insensitive to physiologic noise.

The main findings of the present study were the following: 1) reduced activation in the left lower lateral and the right higher medial somatosensory cortex; 2) decreased cortical surface area in the right BAs 9 and 45 and the left BA 19 and decreased cortical volume in BAs 9 and 10; and 3) reduced FA in major WM tracts.

Histologic studies have demonstrated that 12-24hours after the occurrence of GMH, the proliferation of germ cells in the ganglionic eminence is arrested for up to 4 weeks. ${ }^{9}$ Thrombin and plasmin and toxic neurotransmitters (glutamate and $\gamma$-aminobutyric acid) have been implicated in the pathogenesis of this damage. ${ }^{9}$ Further changes related to GMH-IVH are the presence of extracellular hemoglobin in the periventricular WM and microglia activation. ${ }^{5-7,10}$ Extracellular hemoglobin is rapidly oxidized from the ferrous state to the highly reactive ferric state, which, by activating cytotoxic, oxidative, and inflammatory pathways, may lead to tissue damage. ${ }^{5,7,9,10}$ Damage to the cerebellum has been previously reported after low-grade IVH and has been considered due to the effects of blood products on the superficial granular layer, which represents a germinal matrix. ${ }^{20}$ During the third trimester of gestation, brain microglia are at their highest levels, and after activation, they generate free radicals, secrete cytokines, and promote excitotoxicity. ${ }^{5,7}$ The end result might be injury of the premyelinating oligodendrocytes, axons, subplate neurons, and late-migrating GABAergic neurons present in the WM of very preterm infants. ${ }^{5,7}$ The damage detected depends on the regional developmental stage of the GM and WM at the peak period of GMH-IVH and at the time of MR imaging.
The present study demonstrated decreased AALFF in the right and left somatosensory cortex (SSC). The functional development of the SSC is strongly associated with thalamic input through thalamocortical fibers and depends on thalamic, axonal, and cortical maturational integrity. GMH deprives the thalamus of the latemigrating GABAergic interneurons, while oxidative stress may affect thalamocortical fibers destined for the SSC at a period of maximum developmental vulnerability, which is 1-2 weeks earlier than that of fibers directed to the frontal and occipital association cortex. ${ }^{21}$ The lower fALFF in the left lower lateral and right higher medial SSC is probably related to the right-left thalamic and SSC and rostral-caudal SSC developmental hierarchy. ${ }^{22,23}$ The right thalamus and the right SSC develop before the left, and the lower lateral, before the higher medial SSC. ${ }^{22,23}$ Abnormal fALFF in areas assigned to the face on the left and to the feet on the right SSC, is probably related to the coincidence of the adverse effects with the higher developmental activity and thus the increased vulnerability of these areas. ${ }^{24,25}$ Sensory processing disorder has been reported in $39 \%-52 \%$ of children born preterm, with a higher incidence in those born earlier than 32 weeks' GA. Sensory processing disorder comprises difficulties in interpreting and using sensory information from the environment, resulting in hypo- or hyper-reaction to sensory stimuli. ${ }^{26}$ The results of the present study suggest that decreased resting-state activity in the SSC of very premature infants with low-grade GMH-IVH might herald the future development of sensory processing impairment. Follow-up of these children would be useful to evaluate this hypothesis.

A decrease in total cortical GM volume has been reported in very premature infants with low-grade $\mathrm{IVH}^{13}$ In the present study, local decreases in surface area and volume were detected. During the third trimester, cortical surface area increase is a prominent process, and it has been associated with the gyration process. A disproportionate increase in the superficial cortical layers (II and III) results in cortical folding. ${ }^{1,7}$ BAs 9, 10, and 45 belong to the dorsolateral, medial, and anterolateral prefrontal cortex, which present a protracted developmental window. $\mathrm{GMH}$, by arresting proliferation of the progenitors and by intervening with axonal development, deprives the prefrontal cortex of the late GABAergic neurons and axons and thus impairs cortical maturation.

The right prefrontal cortex is more mature than the left at around term; its developmental vulnerability depends on the GA, and the effects of GMH-IVH probably coincide with the highest developmental activity of these areas; this feature explains the findings of this study. ${ }^{12}$ The prefrontal cortex is associated with higher level cognitive functions, including organization of input from sensory modalities, maintenance of attention, monitoring of information in the working memory, coordination of goaldirected behaviors, emotional processing, sociality, linguistic 
sequencing, planning, syntax, and phonological processing. ${ }^{27}$ An increased incidence of cognitive problems, autism, schizophrenia, and difficulties with complex language functions have been reported in subjects born prematurely, especially those born very preterm. $^{26,27}$

BA 19 belongs to the prestriate cortex, which is a visualassociation cortex, and in contrast to the primary visual cortex, it receives afferents predominantly from the pulvinar of the thalamus. ${ }^{28}$ The left prestriate cortex develops before the right, and GMH may result in decreased numbers of late-migrating GABAergic neurons, which could explain the decreased cortical surface area demonstrated here. Cognitive visual dysfunction is a spectrum of abnormalities, ranging from neurosensory impairment to higher order deficits in visual perception, visual attention, and visuospatial working memory, and it is one of the common abnormalities in children born prematurely. ${ }^{29}$ Overall, the cortical developmental abnormalities reported in this study suggest that low-grade IVH may be associated with these morbidities.

The present study demonstrated decreased FA in major projection, commissural, and association WM tracts. During the third trimester, WM, though unmyelinated, presents with anisotropy related to ensheathment of axons by premyelinating oligodendrocytes, decreases in the extra-axonal space due to water reduction, and increases in fiber diameter and axoplasmic flow. ${ }^{7,30}$ IVH, by arresting the development of oligodendroglia precursor cells and by injuring premyelinating oligodendrocytes and axons, may lead to a decrease in FA. FA derives from the SD of the 3 eigenvalues $(\lambda 1, \lambda 2, \lambda 3)$ and thus depends on axial diffusivity and radial diffusivity. ${ }^{31}$ Disproportionate changes in axial diffusivity and radial diffusivity, even to a degree not reaching statistical significance, may affect FA and lead to significant changes. One previous study in premature infants with low-grade $\mathrm{IVH}^{14}$ demonstrated an isolated decrease of FA in major WM tracts in infants with a GA $>29$ weeks, in agreement with the present study. Some differences in the affected WM tracts are probably related to the different GAs of the infants included in the 2 studies. ${ }^{14}$

Low-grade IVH has been associated with a 2 -fold increase in the risk of lower cognitive performance and a 2.6-fold increase in the risk of neuromotor abnormalities. ${ }^{4,32}$ Most of the WM tracts implicated in the present study have been associated with these functions. Specifically, the superior longitudinal fasciculus is involved in the processes of attention, memory, and emotion; the inferior longitudinal fasciculus and the inferior fronto-occipital fasciculus are associated with the thought process, visual emotion, and cognition; the uncinate fasciculus is critical in emotion and memory; the anterior thalamic radiation is associated with mood regulation; and the cingulum, with emotion, reward, and memory. ${ }^{18,22}$ Previous studies have reported that preterm infants with severe IVH are at risk of developing spastic diplegia, and damage to the corticospinal tracts traveling in the periventricular matter is considered the underlying cause. ${ }^{33}$ Our findings are in agreement with a previous study in very premature infants with lowgrade IVH, which demonstrated low FA of the corticospinal tracts, ${ }^{34}$ suggesting that the corticospinal tracts may also be affected by low-grade IVH.
This study has some limitations, primarily the small sample size, which does not allow correlation with neurodevelopmental data, weakens the statistical power, and possibly masks differences among important clinical confounders. Additionally, the lowangular-resolution DTI cannot handle adequately the "crossingfiber" problem or the non-Gaussian nature of diffusion. Larger cohorts and more advanced diffusion protocols are required to extend and validate this study.

\section{CONCLUSIONS}

The present study shows that at around term-equivalent age, very premature infants with low-grade IVH may present imaging findings of regional abnormalities of the brain, appearing as cortical underdevelopment, functional impairment, and microstructural immaturity of major WM tracts.

\section{REFERENCES}

1. Raybaud C, Ahmad T, Rastegar N, et al. The premature brain: developmental and lesional anatomy. Neuroradiology 2013;55(Suppl 2): 23-40 CrossRef Medline

2. Papile LA, Burstein J, Burstein R, et al. Incidence and evolution of subependymal and intraventricular hemorrhage: a study of infants with birth weights less than 1,500 gm. J Pediatr 1978;92:529-34 CrossRef Medline

3. Mukerji A, Shah V, Shah PS. Periventricular/intraventricular hemorrhage and neurodevelopmental outcomes: a meta-analysis. Pediatrics 2015;136:1132-43 CrossRef Medline

4. Inder TE. Neurodevelopmental impact of low-grade intraventricular hemorrhage in very preterm infants. J Pediatr 2006;149:152-54 CrossRef Medline

5. Volpe JJ. Impaired neurodevelopmental outcome after mild germinal matrix-intraventricular hemorrhage. Pediatrics 2015;136:118587 CrossRef Medline

6. Volpe JJ. Brain injury in premature infants: a complex amalgam of destructive and developmental disturbances. Lancet Neurol 2009;8: 110-24 CrossRef Medline

7. Volpe JJ. The encephalopathy of prematurity-brain injury and impaired brain development inextricably intertwined. Semin Pediatr Neurol 2009;16:167-78 CrossRef Medline

8. McAllister JP, Guerra MM, Ruiz LC, et al. Ventricular zone disruption in human neonates with intraventricular hemorrhage. $J$ Neuropathol Exp Neurol 2017;76:358-75 CrossRef Medline

9. Del Bigio MR. Cell proliferation in human ganglionic eminence and suppression after prematurity-associated haemorrhage. Brain 2011;134:1344-61 CrossRef Medline

10. Ley D, Romantsik O, Vallius S, et al. High presence of extracellular hemoglobin in the periventricular white matter following preterm intraventricular hemorrhage. Front Physiol 2016;7:330 CrossRef Medline

11. Tamnes CK, Herting MM, Goddings AL, et al. Development of the cerebral cortex across adolescence: a multisample study of interrelated longitudinal changes in cortical volume, surface area, and thickness. J Neurosci 2017;37:3402-12 CrossRef Medline

12. Mouka V, Drougia A, Xydis VG, et al. Functional and structural connectivity of the brain in very preterm infants: relationship with gestational age and body and brain growth. Pediatr Radiol 2019; 49:1078-84 CrossRef Medline

13. Vasileiadis GT, Gelman N, Han VK, et al. Uncomplicated intraventricular hemorrhage is followed by reduced cortical volume at near-term age. Pediatrics 2004;114:e367-72 CrossRef Medline

14. Tortora $D$, Martinetti $C$, Severino $M$, et al. The effects of mild germinal matrix-intraventricular haemorhage on the developmental 
white matter microstructure of preterm neonates: a DTI study. Eur Radiol 2018;28:1157-66 CrossRef Medline

15. Zollei L, Martinos A. FreeSurfer image processing pipeline for infant clinical MRI images. In: Proceedings of the 23rd Annual Meeting of the Organization for Human Brain Mapping, Vancouver, British Columbia, Canada; June 25-29, 2017

16. Van Essen DC. A Population-Average, Landmark- and SurfaceBased (PALS) atlas of human cerebral cortex. Neuroimage 2005;28: 635-62 CrossRef Medline

17. Ball G, Counsell SJ, Anjari M, et al. An optimised tract-based spatial statistics protocol for neonates: applications to prematurity and chronic lung disease. Neuroimage 2010;53:94-102 CrossRef Medline

18. Zou QH, Zhu CZ, Yang Y, et al. An improved approach to detection of amplitude of low-frequency fluctuation (ALFF) for restingstate fMRI: fractional ALFF. J Neurosci Methods 2008;172:137-41 CrossRef Medline

19. Papageorgiou I, Astrakas LG, Xydis V, et al. Abnormalities of brain neural circuits related to obesity: a diffusion tensor imaging study. Magn Reson Imaging 2017;37:116-21 CrossRef Medline

20. Tam EW, Miller SP, Studholme C, et al. Differential effects of intraventricular hemorrhage and white matter injury on preterm cerebellar growth. J Pediatr 201 1;158:366-71 CrossRef Medline

21. Krsnik Z, Majic V, Vasung L, et al. Growth of thalamocortical fibers to the somatosensory cortex in the human fetal brain. Front Neurosci 2017;11:233 CrossRef Medline

22. Whitehead K, Papadelis C, Laudiano-Dray MP, et al. The emergence of hierarchical somatosensory processing in late prematurity. Cereb Cortex 2019;29:2245-60 CrossRef Medline

23. Tzarouchi LC, Astrakas LG, Xydis V, et al. Age-related grey matter changes in preterm infants: an MRI study. Neuroimage 2009;47: 1148-53 CrossRef Medline

24. Kostovic I, Judas M. The development of the subplate and thalamocortical connections in the human foetal brain. Acta Paediatr 2010;99:1119-27 CrossRef Medline
25. Dall'Orso S, Steinweg J, Allievi AG, et al. Somatotopic mapping of the developing sensorimotor cortex in the preterm human brain. Cereb Cortex 2018;28:2507-15 CrossRef Medline

26. Ryckman J, Hilton C, Rogers C, et al. Sensory processing disorder in preterm infants during early childhood and relationships to early neurobehavior. Early Hum Dev 2017;113:18-22 CrossRef Medline

27. Teffer K, Semendeferi K. Human prefrontal cortex: evolution, development, and pathology. Prog Brain Res 2012;195:191-218 CrossRef Medline

28. Kostovic I, Rakic P. Development of prestriate visual projections in the monkey and human fetal cerebrum revealed by transient cholinesterase staining. J Neurosci 1984;4:25-42 CrossRef Medline

29. Ceschin R, Wisnowski JL, Paquette LB, et al. Developmental synergy between thalamic structure and interhemispheric connectivity in the visual system of preterm infants. Neuroimage Clin 2015;8:46272 CrossRef Medline

30. Suzuki Y, Matsuzawa H, Kwee IL, et al. Absolute eigenvalue diffusion tensor analysis for human brain maturation. NMR Biomed 2003;16:257-60 CrossRef Medline

31. Jellison BJ, Field AS, Medow J, et al. Diffusion tensor imaging of cerebral white matter: a pictorial review of physics, fiber tract anatomy, and tumor imaging patterns. AJNR Am J Neuroradiol 2004;25:356-69 Medline

32. Patra K, Wilson-Costello D, Taylor HG, et al. Grades I-II intraventricular hemorrhage in extremely low birth weight infants: effects on neurodevelopment. J Pediatr 2006;149:169-73 CrossRef Medline

33. Linsell L, Malouf R, Morris J, et al. Prognostic factors for cerebral palsy and motor impairment in children born very preterm or very low birthweight: a systematic review. Dev Med Child Neurol 2016;58:554-69 CrossRef Medline

34. Morita T, Morimoto M, Yamada K, et al. Low-grade intraventricular hemorrhage disrupts cerebellar white matter in preterm infants: evidence from diffusion tensor imaging. Neuroradiology 2015;57:507-14 CrossRef Medline 